\title{
Survol et bilan de la microchirurgie andrologique "Un baroud d'honneur" pour une cause perdue
}

\author{
SchoysmaN R.
}

S.I.M.A.F. Hôpital Van Helmont, Vaarstraak, 42, B.1800 - VILVOORDE, Belgique

\section{RESUME}

Les diverses techniques de chirurgie épididymaire ainsi que leurs résultats sont passés en revue. Il est insisté sur la sélection pré-opératoire des cas. Ces résultats sont comparés à ceux des techniques de microinsémination de spermatozoïdes épididymaires ou testiculaires. Le bilan des deux approches permet de maintenir une position chirurgicale dans $40 \%$ des cas d'oblitération épididymaire, les résultats en grossesses étant équivalents à ceux de la PMA, mais à un coût beaucoup moindre tant pour le patient que pour la société.

Mots clés : Microchirurgie, Anastomoses épididymaires, Assistance médicale à la procréation.

\section{INTRODUCTION}

La pathologie épididymaire responsable d'une oblitération partielle ou totale $d u$ transit spermatique est diverse. Certaines lésions sont extérieures à l'épididyme lorsqu'il est étranglé par des adhérences serrées au point que le transit y est interrompu. D'autres sont provoquées par des formations kystiques qui peuvent également mener à un certain degré d'oblitération. Les causes les plus fréquentes, cependant, sont des séquelles post-infectieuses dont les agents sont bien connus. En premier lieu, il y a la gonorrhée, suivie et même dépassée, à l'heure actuelle, par les infections à Chlamydia. Les lésions qui résultent des infections soit blennorragiques soit à Chlamydia ne sont pas identiques. La blennorragie mène généralement à l'azoospermie totale par une oblitération localisée au niveau de la queue épididymaire. La Chlamydia, par contre, lèse l'épididyme sur toute sa longueur, sans être nécessairement totalement obstructive. En fait, un bon nombre d'oligospermies, dues à des oblitérations épididymaires partielles, sont à tort interprétées comme étant d'origine testiculaire. Dans le survol et le bilan envisagé ici, nous ne prendrons en considération que les oblitérations totales et leur traitement par anastomose épididymo-déférentielle.

\section{LES TECHNIQUES}

Au début de ce siècle l'agent pathogène oblitérant l'épididyme était principalement le gonocoque. Les premiers cliniciens qui se sont penchés sur la correction des oblitérations étaient l'américain Martin et le russe Ruzumovski. Ils avaient compris l'intérêt de pratiquer un court-circuitage entre la partie encore saine de l'épididyme et le canal déférent, mais ces premières tentatives furent évidemment couronnées de peu de succès. A partir des années 30, l'américain $\mathrm{O}^{\prime}$ Connor et le français $\mathrm{H}$. Bayle se sont particulièrement intéressés à cette pathologie et ont opéré pendant vingt ans un grand nombre de patients par anastomo- 
se latéro-latérale. Elle consistait à ouvrir la tête épididymaire longitudinalement, en amont de l'obstacle cliniquement reconnu, de pratiquer une incision équivalente dans le canal déférent et de réunir ces deux ouvertures par une suture latéro-latérale à l'aide d'un nombre variable de points au fil tentale. Bayle s'efforçait de disséquer à la loupe des granulomes (qu'il appelait spermatorrhages) bien reconnaissables à l'intérieur de la tête épididymaire. D'autres chirurgiens se sont progressivement intéressés à cette pathologie et l'ensemble de leurs résultats est repris dans le Tableau 1.

Vers le milieu des années soixante-dix, cette approche apparaissait cependant décevante. Le taux de succès ne dépassait pas les 25 à $30 \%$ en perméabilité et le taux de grossesses arrivait à peine à $15 \%$. Parmi les efforts pour améliorer ces résultats, il y a celui de Schoysman qui incluait, dans l'angle supérieur de l'anastomose latéro-latérale, un tubule épididymaire incisé, ce qui offrait une légère amélioration dans les résultats. Mais la vraie chirurgie épididymaire commencait avec les contributions de Owen et Silber. En un premier temps, ils préconisaient de sectionner l'épididyme de part en part en amont de l'obstacle recon- nu, de rechercher dans la tranche de section le tubule dont des spermatozoïdes continuaient de s'écouler, puis de sectionner à angle droit le canal déférent et de pratiquer une anastomose termino-terminale entre le tubule épididymaire sélectionné et la muqueuse du canal déférent. L'intervention est alors complétée par la suture du pourtour de la section épididymaire et celle du déférent. Cette technique était de toute évidence beaucoup plus précise et les résultats ne se sont pas fait attendre puisque les taux de récupération de perméabilité et, dès lors, de grossesses étaient nettement plus favorables qu'après l'ancienne anastomose latéro-latérale.

Cependant, cette approche offrait des difficultés, à savoir que le diamètre du canal déférent ne permettait pas toujours une coaptation avec le diamètre de l'épididyme sectionné. En effet, il fallait parfois sectionner l'épididyme de plus en plus près du rete testis, en un endroit où son diamètre est plus grand et il devenait de plus en plus difficile, voire impossible d'obtenir une bonne coaptation. Aussi, cette intervention fut-elle remplacée par une anastomose latéro-terminale qui consiste à libérer quelques tubules épididymaires, d'en inciser un pour

Tableau 1 : Résultats des anastomoses latéro-latérales macrochirurgicales.

\begin{tabular}{lcccc}
\hline & Années & Nombre de cas & Perméabilité & Grossesses \\
\hline Jequier & 1985 & 24 & $3(12.5 \%)$ & $1(4.2 \%)$ \\
Hanley & 1955 & 71 & $11(15,5 \%)$ & $5(7 \%)$ \\
Dubin & 1984 & 68 & $32(47 \%)$ & $11(16 \%)$ \\
O'Conor & 1953 & 23 & $14(61 \%)$ & $5(22 \%)$ \\
Lee & 1978 & 82 & $25(30.4 \%)$ & $11(13.4 \%)$ \\
Wagenknecht & 1980 & 170 & $48 \% 32 \%$ & - \\
Hendry & 1983 & 83 & $29(35 \%)$ & $9(10.8 \%)$ \\
Kar & 1975 & 281 & $137(48.8 \%)$ & $40(14.2 \%)$ \\
Schoysman & 1981 & 261 & $146(56 \%)$ & $21 \%$ \\
Hendry & 1981 & 5 & $3(60 \%)$ & - \\
Schoysman & 1986 & 565 & $350(62 \%)$ & $18 \%$ \\
Hagner & 1936 & 33 & $21(64 \%)$ & 16 \\
Bayle & 1952 & 65 & $43(66 \%)$ & $20(30.7 \%)$ \\
Pontonnier & 1984 & 67 & 27 & $9(13 \%)$ \\
Cognat & 1975 & 56 & & $9 \%$ \\
\hline
\end{tabular}


y vérifier la présence de spermatozoïdes et de pratiquer une anastomose entre cette incision tubulaire et la muqueuse déférentielle. C'est cette dernière intervention, dite latéro-terminale, qui est à ce jour la plus pratiquée, avec les résultats favorables comme il apparaît dans le Tableau 2.

\section{LES ALEAS DES TENTATIVES DE VASO-EPIDIDYMOSTOMIES}

Tout patient azoospermique avec bilan hormonal normal et examen clinique suggérant une oblitération de l'épididyme n'est pas pour autant un bon candidat pour une anastomose épididymo-déférentielle. En effet, plusieurs difficultés peuvent surgir. Il n'est pas rare qu'à l'oblitération épididymaire soit associée une séquelle de déférentite. Le patient présente alors deux obstacles sur ses voies séminales, à savoir celui de l'épididyme et celui du canal déférent, jusqu'y compris le canal éjaculateur. Cette complication supplémentaire se retrouve dans $8 \%$ des cas (Tableau 3 ).

Une autre difficulté est une spermatogénèse beaucoup moins favorable que les dosages hormonaux ne le laissent prévoir. En effet, même lorsque la FSH est normale, la spermatogénèse peut être très déficiente et n'atteindre que le stade de spermatocyte de deuxième ordre ou de spermatide. La dissection des tubules épididymaires et l'examen de leur contenu ne permettent, dès lors, pas de mettre des spermatozoïdes en évidence. Enfin, une troisième difficulté réside dans le fait que l'épididyme peut cliniquement paraître augmenté de volume, mais l'exploration de visu, et surtout sous microscope opératoire, montre que la tête n'est pas gorgée de spermatozoïdes mais est en fait une structure scléreuse dans laquel-

Tableau 2 : Résultats des anastomoses microchirurgicales.

\begin{tabular}{|c|c|c|c|c|}
\hline & Années & Nombre de cas & Perméabilité & Grossesses \\
\hline McLoughlin & 1982 & 23 & - & $9(39 \%)$ \\
\hline Wagenknecht & 1985 & 50 & - & $12(23 \%)$ \\
\hline Dubin & 1984 & 46 & $18(39 \%)$ & $6(13 \%)$ \\
\hline Thomas & 1987 & 50 & $33(66 \%)$ & $18(41.9 \%)$ \\
\hline Schoysman & 1990 & 161 & $126(78 \%)$ & $\begin{array}{c}67(41 \% \text { s/total }) \\
\text { (53\% s/perméables })\end{array}$ \\
\hline Fogdestam & 1986 & 41 & $35(85.3 \%)$ & $15(36.6 \%)$ \\
\hline Silber & 1978 & 14 & $12(86 \%)$ & - \\
\hline Belgrano & 1984 & 4 & $4(100 \%)$ & $1(25 \%)$ \\
\hline \multicolumn{5}{|l|}{ Belker } \\
\hline first group & & 12 & $1(8 \%)$ & $0(0 \%)$ \\
\hline latest group & & 12 & $9(75 \%)$ & $3(25 \%)$ \\
\hline Goldstein & & 25 & $15(60 \%)$ & $3(12 \%)$ \\
\hline Sharlip & & 20 & $8(40 \%)$ & $5(25 \%)$ \\
\hline \multirow[t]{3}{*}{ Silber } & 1989 & 541 & & \\
\hline & & 77 caput & $81 \%$ & $26 \%$ \\
\hline & & 464 corpus & $91 \%$ & $59 \%$ \\
\hline Lee & 1985 & 62 & $21(36 \%)$ & $12(21 \%)$ \\
\hline \multirow[t]{4}{*}{ Wagenknecht } & & 327 & & \\
\hline & & $\uparrow$ caput & $11 \%$ & $0 \%$ \\
\hline & & $\downarrow$ caput & $47 \%$ & $32 \%$ \\
\hline & & corpus & $76 \%$ & $52 \%$ \\
\hline Owen & 1982 & 60 & & $19(31 \%)$ \\
\hline Matsuda & 1993 & 24 & $80 \%$ & $9(43 \%)$ \\
\hline
\end{tabular}


a) L'examen clinique montre des têtes

épididymaires rétractées \& indurées

b) Cicatrisation +++ après chirurgie préalable
$25 \%$

(

\section{Exploration chirurgicale $75 \%$}

$\begin{array}{lll}30 \% \text { écoulement très réduit ou nul } & \begin{array}{l}\text { oblitération } \\ \text { déférentielle } \\ \text { associée }\end{array} & 8 \%\end{array}$

Biopsie testiculaire défavorable

Cas favorables pour chirurgie $37 \%$

$23 \%$ situation favorable

canal perméable

spermes présents dans l'épididyme
Situation idéale
canal perméable
oblitération
épididymaire basse
sperme abondant et
même mobile

$14 \%$

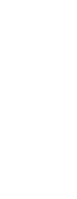

le la dissection la plus poussée ne permet pas de retrouver des tubules contenant des spermatozoïdes. Ce sont principalement les lésions provoquées par la Chlamydia qui peuvent prendre ces formes. Dans certains cas extrêmes, on ne retrouve des spermatozoïdes que dans les tubules très proches du rete testis, c'est à dire au niveau même d'un des canaux efferents testiculaires. Ceci a donné lieu à certaines approches microchirurgicales véritablement acrobatiques dont les résultats sont aléatoires.

Il découle de toutes ces considérations que toute azoospermie excrétoire n'est évidemment pas curable et que seule la moitié des patients peuvent bénéficier d'une intervention de microchirurgie. Il n'en reste pas moins que les cas opérables sont nombreux et ont donné lieu à un grand nombre de succès. Leur analyse a été reprise dans divers travaux et étudiée sous l'angle non seulement de la technique d'anastomose, mais également de la qualité du sperme recueilli lors de l'ouverture des tubules, de la qualité de la spermatogénèse et de la durée d'existence de l'oblitération s'il était possible de la déterminer. Il faut reconnaître que les microchirurgiens épididymaires qui ont publié de bonnes séries de cas ne sont pas particulièrement nombreux et leur nombre ne dépasse pas deux douzaines.

Depuis 1985, une nouvelle arme a été utilisée dans les stérilités épididymaires. Elle consiste à prélever des spermatozoïdes dans l'épididyme en amont de l'obstacle, et de les utiliser dans des programmes de fécondation médicalement assistée. En un premier temps, cette technique fut utilisée en cas d'agénésie des canaux déférents. 
Elle s'est cependant rapidement étendue à des oblitérations post-infectieuses non corrigibles par microchirurgie ou encore à des échecs de reperméabilisation de vasectomie.

En un premier temps, l'usage de spermatozoïdes épididymaires, que ce soit en cas d'agénésie déférentielle ou en cas de séquelles post-infectieuses, n'était pas particulièrement prometteur. Les résultats des PMA avec sperme épididymaire et insémination ovocytaire classique menaient à des taux de grossesses fluctuant entre $5 \& 10 \%$. Plus récemment les micro-inséminations de spermatozoïdes, d'abord par SUZI et ensuite par ICSI en ont modifié le pronostic de façon très significative. En effet, l'ICSI avec insémination de spermatozoïdes épididymaires (technique dite MESA) mène à des taux de grossesses beaucoup plus élevés que par insémination classique.

Dans ce domaine il y a également des surprises, en ce sens que la tête épididymaire, parfois réduite à un petit nodule, ne contient aucun spermatozoïde. En 1992, nous avons dans un de ces cas utilisé pour la première fois des spermatozoïdes récoltés directement dans le testicule. Alors que chez l'animal d'expérience une grossesse n'avait jamais pu être obtenue avec des cellules aussi immatures, il n'en a pas été de même chez l'homme, puisque des fertilisations ont pu être obtenues, suivies de grossesses. Notre propre expérience est résumée dans le Tableau 4. D'autres équipes se sont intéressées à cette idée et ont confirmé l'intérêt de l'usage de spermatozoïdes testiculaires dans des programmes de PMA (technique TESE).

Dès lors, à l'heure actuelle, une question importante se pose : La chirurgie andrologique est-elle destinée à suivre le destin de la microchirurgie tubaire ? Cette dernière a perdu beaucoup de son intérêt depuis l'utilisation de la PMA. Il serait erroné de dire qu'elle a disparu de l'arsenal thérapeutique destiné à corriger la stérilité tubaire, mais les indications et contre-indications sont à l'heure actuelle bien codifiées, au point que ne sont plus réservés à la chirurgie tubaire que certains cas sélectionnés dont les indications gardent alors toute leur valeur. Il en est particulièrement ainsi pour les réparations de ligatures tubaires.

Il en sera vraisemblablement de même pour la chirurgie de l'épididyme. Tous les cas dans lesquels la tête épididymaire n'est pas gorgée de spermatozoïdes mais qui ont toujours une spermatogénèse normale, auraient vraisemblablement avantage à être repris dans des programmes de PMA avec utilisation de spermatozoïdes testiculaires. Par contre, des oblitérations caudales avec bon engorgement du caput restent d'excellentes indications pour une vaso-épididymostomie latéro-terminale. Tout comme pour la microchirurgie gynécologique, la réparation de vasectomies regrettées reste une excellente indication opératoire.

Tableau 4 : Résultats des MESA \& TESE.

\begin{tabular}{lcc}
\hline & MESA & TESE \\
NOMBRE DE CYCLES & $-\quad 49$ & 43 \\
2 PN / INJECTES PAR OVOCYTE & $55 \%$ & $65 \%$ \\
TRANSFERTS & $100 \%$ & $100 \%$ \\
FAUSSES-COUCHES & $16 \%$ & $20 \%$ \\
GROSSESSES EVOLUTIVES PAR CYCLE & $33 \%$ & $28 \%$ \\
\hline
\end{tabular}


Si d'aucuns ont suggéré que la microchirurgie andrologique avait fait son temps et devait être totalement abandonnée au profit de la PMA avec sperme testiculaire, ils perdent de vue le très important problème financier pour le patient. En effet, refuser à un patient le bénéfice du recouvrement de sa fertilité dans $40 \%$ des cas, pour conseiller d'emblée une micro-insémination ovocytaire, c'est l'entraîner dans une entreprise beaucoup plus onéreuse et pas nécessairement plus favorable en tant que taux de grossesses. Le calcul comparatif des frais chirurgie/PMA est évidemment variable d'un pays à l'autre selon la sécurité sociale. En ce qui concerne la Belgique, pour le patient, le coût d'un seul essai de fécondation in vitro MESA ou TESE équivaut à une microchirurgie. Pour la société un seul essai de PMA est une dépense 3 à 4 fois supérieure à la chirurgie. Il faut, dès lors, que chaque cas soit évalué de la façon la plus précise possible, pour offrir la meilleure approche aux patients souffrant d'azoospermie excrétoire. Une telle évaluation doit nécessairement inclure le volet gynécologique du problème, dans lequel l'âge de la conjointe a toute son importance.

\section{BIBLIOGRAPHIE}

La bibliographie concernant la microchirurgie épididymaire et les techniques de MESA et de TESE est exhaustive.

Sur demande la liste complète sera envoyée.

\section{ABSTRACT \\ Techniques of epididymal surgery and their results \\ R. SchoYsman}

The techniques of epididymal surgery and their results are briefly reexposed and compared to the results of assisted procreation with epididymal or testicular sperm.

Microsurgery of the epididymis remains a very valuable indication in a selected group of patients leading to the same amount of pregnancies after an average of three attempts by microinsemination of oocytes with epididymal sperm. The expense both for patient and society is much in favour of a surgical approach.

Key words : Epididymal surgery, ICSI. 